\title{
Development and Experimental Demonstration of a Plug-and-Play Multiple RTU Coordination Control Algorithm for Small/Medium Commercial Buildings
}

\author{
D. Kim ${ }^{\mathrm{a}, *}$, J.E. Braun ${ }^{\mathrm{a}}$, J. Cai ${ }^{\mathrm{a}}$, D.L. Fugate ${ }^{\mathrm{b}}$ \\ ${ }^{a}$ School of Mechanical Engineering, Purdue University, West Lafayette, IN, USA \\ ${ }^{b}$ Oak Ridge National Laboratory, Oak Ridge, TN, USA
}

\begin{abstract}
There have been very few advanced control algorithms developed and deployed for small/medium commercial buildings due to practical difficulties such as the lack of Building Management System (BMS), significant disturbances, high sensor costs and high cost of site-specific engineering solutions. These high implementation costs have been a major impediment to successful market penetration. The focus of this work is to develop and demonstrate a practical control algorithm for the coordination of multiple roof top units (RTUs). The goal is to minimize sensor and configuration requirements in order to enable a more cost effective control implementation for small/medium commercial building applications. The algorithm characterizes the responses of the thermostat sensors to RTU cycling and uses a model within an optimization algorithm to determine the best combination of RTUs to operate in order to minimize power and maintain comfort conditions over a short prediction horizon. The algorithm was first tested in simulation for an existing sit-down restaurant and then at a field site that is a gymnasium. The estimated energy savings were greater than $20 \%$ for the restaurant and about $8 \%$ for the gym. Peak demand savings of about $40 \%$ were demonstrated for the gym. The algorithm is termed "plug-and-play" because no additional sensors are needed for implementation and the thermostat response models and optimization require minimal configuration.
\end{abstract}

Keywords: RTU coordination, Model predictive control, Advanced building control, Small/medium building control

\section{Introduction}

Buildings are responsible for about $40 \%$ of the total primary energy usage in the US. In the past three decades, various advanced control approaches

\footnotetext{
${ }^{*}$ Corresponding author

Email address: kim1077@purdue.edu, tel: 1-765-586-6076 (D. Kim)
}

Preprint submitted to Energy and Building

August 7, 2015 
have been studied to reduce energy consumption especially for large commercial buildings. One of the most popular and widely studied approaches is Model Predictive Control (MPC), in which an optimal control problem over a finite prediction horizon is solved at each sampling time using a system model. Many papers show the capability of MPC to reduce energy consumption (Ma et al., 2010; Prívara et al., 2011) and demand cost (Braun, 1990). MPC would typically be integrated within a Building Management System (BMS) where real-time measured data are stored. The control algorithm relies on future predictions of disturbances ${ }^{1}$ such as outdoor air temperature and solar radiation.

However there have been very few advanced control algorithms developed for many small/medium commercial buildings, such as retail stores, restaurants and small businesses, where multiple roof top units (RTUs) are utilized to provide heating and cooling for open spaces. This is mainly because such buildings typically do not have a BMS system and it would be expensive to add the required sensors.

Furthermore, large open spaces served by multiple RTUs can pose significant control challenges, such as spatial comfort variations and short-cycling of units. A conventional approach for this application relies on local feedback control, where each RTU is cycled on and off using its own thermostat that is located in the vicinity of its supply diffusers in the open space. This can lead to poor coordination among the RTUs where some units carry the majority of the load, some units cycle on and off very frequently and others operate infrequently.

The focus of this work is to develop and demonstrate a practical model-based control algorithm for ON/OFF staging of multiple RTUs. The control approach was designed to minimize sensor and configuration requirements in order to enable a more cost effective control implementation for small/medium commercial building applications. The minimal sensor requirement is the unique feature of the presented algorithm which enables to reduce its capital and maintenance cost, hence enables to broaden its application range especially for buildings that have no BMS. The controller aims at reducing the energy consumption and the electric peak demand cost with low sensor requirements for multiple RTU coordination. The simulation and experimental results show that significant energy savings/demand reduction potential exists and the savings can be achieved purely by the coordination control. The overall procedure from modeling to controller design and the simulation and experimental results are provided in the paper.

\section{System Description and RTU coordination}

Fig. 2a and 8a show example buildings served by multiple RTUs that are used as case studies in this paper. These types of buildings served by multipleRTUs can be characterized as follows.

\footnotetext{
${ }^{1}$ Any uncontrollable inputs to a system are called disturbances.
} 
- Several RTUs serve one big open-space. Therefore there is unknown but possibly significant inter-zonal convective coupling, namely the state of one zone affecting the dynamics of neighboring zones.

- Various supply air duct systems and different performance characteristics for multiple RTUs.

- Only a few integer modes/stages are allowed to control each RTU. For example, only the selection between 0 and 1 is feasible for controlling a single-speed compressor RTU where the modes correspond to ON/OFF status of the compressor.

To formulate the problem, let $p, m \in \mathbb{N}$ be the number of thermostats and the total number of RTU stages, respectively. The manipulated variables are the RTU stages, denoted as $u \in \mathbb{N}^{m}$, and the controlled variables are the thermostat temperatures, denoted as $y \in \mathbb{R}^{p}$. The system, $G: u \mapsto y$, in nature is very complex. This is because it involves a refrigerant cycle, the heat exchange between ventilation air and the refrigerant, inter-zonal air flows that could vary depending on the combination of the supply air fan modes and the time constants of thermostats. It is reasonable to choose a black box modeling approach to deal with these complexities.

The main challenges for applying system identification methods for small and medium-sized commercial buildings are;

- The lack of BMS

- Unmeasured disturbances and their correlation to the control inputs when open-loop experiments are not feasible due to practical issues

- Large numbers of the controlled variables

- Time varying system properties, e.g. performance degradation of RTUs

For these type of systems, the available information are the control inputs/outputs. When weather information, e.g. the outdoor air temperature and the solar radiation, is not available, it is described as unmeasured disturbances.

Because of the difficulties, the first target is to get a crude approximating model for the true system relying only on the thermostat temperatures and the stages of RTUs without any disturbance information. Then, the next step is to adjust the estimated model in order to compensate for the system-model mismatch and the unmodeled disturbances. Detailed descriptions of the algorithms will be presented in Section 3.

Before describing the algorithm details, it is worth clarifying distinctions between RTU control and RTU coordination that is discussed in this paper. RTU control concerns how to make a single RTU operate more efficiently and includes control logic for fan $\mathrm{ON} / \mathrm{OFF}$, fan speeds and outdoor air damper positions. Therefore, Advanced Rooftop Control (ARC) consists of integrated 
air-side economizer controls, supply-fan speed controls, cooling capacity controls and demand-controlled ventilation controls (Wang et al., 2013). The ARC algorithms use each thermostat's cooling/heating demand signals determined by cooling/heating set points.

RTU coordination consists of choosing how to coordinate multiple RTUs to operate more efficiently as a group or system. It focuses not on a single unit, rather on a group of RTUs that serve one building. The cooling/heating demand signals, which are inputs for the individual RTU control, are the variables for decision making. Due to the hierarchy, a RTU coordinator is a supervisory controller while a RTU controller can be viewed as a local controller (See Fig. 1). The energy benefits of the coordination are more favorable for buildings that have multiple RTUs with different efficiencies, which may be the result of RTUs with different rated efficiencies being replaced at different times or the performance of existing RTUs degrading at the different rates due to uneven loading.

Recently, a RTU transactional network has been developed that uses the Volttron Platform developed by Pacific Northwest National Laboratory (PNNL, U.S.), Lawrence Berkeley National Laboratory (LBNL, U.S.), and Oak Ridge National Laboratory (ORNL, U.S.). This platform facilitates the implementation of applications, such as advanced controls/coordination and automated fault detection and diagnostics (AFDD) and may be viewed as an interface between hardware, e.g. sensors and compressors, and software applications. Katipamula et al. (2013) developed a demand response agent for this platform that controls individual RTU using a rule-based algorithm. More recently, Nutaro et al. (2014) developed a low cost retrofit RTU coordination algorithm and implemented it within Volttron. This method combines an input-output model with heuristics and optimization to limit peak demand while maintaining comfort. The current work differs in several respects, including a focus on energy minimization, inclusion of a formal optimization algorithm, and use of a different model development approach.

\section{Construction of Supervisory RTU coordinator}

Our goal in this paper is to develop a supervisory coordination algorithm for small/medium size buildings with the objective of minimizing energy consumption, reducing electrical peak demand, and short cycling, while maintaining comfort. This section describes the overall procedure for developing the RTU coordination controller starting from a modeling approach.

\subsection{Modeling and Parameter Estimation Approach}

We chose the least-squares (LS) identification algorithm (see Ljung (1999) for the algorithm details) with an auto-regressive with exogenous input (ARX) model structure. The multi-input and multi-output (MIMO) ARX model has the following general form. 


$$
\begin{aligned}
A\left(z^{-1}\right) y(k) & =B\left(z^{-1}\right) u(k)+\epsilon(k) \\
A\left(z^{-1}\right) & =I+A_{1} z^{-1}+\cdots+A_{n_{a}} z^{-n_{a}}, \\
B\left(z^{-1}\right) & =B_{1} z^{-1}+\cdots+B_{n_{b}} z^{-n_{b}},
\end{aligned}
$$

where $z^{-1}$ is the backward shift operator such that $z^{-1} x(k)=x(k-1) . y(k) \in$ $\mathbb{R}^{p}$ and $u(k) \in \mathbb{R}^{m}$ are the outputs and inputs, respectively. $\epsilon(k) \in \mathbb{R}^{p}$ is a zero mean white noise process. The matrices of $A_{j} \in \mathbb{R}^{p \times p}$ for $j \in\left\{1, \cdots, n_{a}\right\}$ and $B_{i} \in \mathbb{R}^{p \times m}$ for $i \in\left\{1, \cdots, n_{b}\right\}$ are the parameters to be determined based on input/output (I/O) data. $\left(n_{a}, n_{b}\right)$ are the degrees of matrix polynomials, $A\left(z^{-1}\right), B\left(z^{-1}\right)$. We denote an ARX model with degree of $\left(n_{a}, n_{b}\right)$ as $\operatorname{ARX}\left(n_{a}, n_{b}\right)$.

Assume parameters, $\Theta:=\left[A_{1}, \ldots, A_{n_{a}} \mid B_{1}, \ldots, B_{n_{b}}\right]$, were obtained from I/O data by the LS method. For the RTU coordination, we are interested in several step ahead predictions under candidate inputs. For example, suppose we are interested in the optimal $d$-step ahead prediction of the output $y(k+d)$, given past historical data, $y(k), y(k-1), \cdots, u(k-1), u(k-2), \cdots$ and given candidate current and future inputs, $u(k+d-1), u(k+d-2), \cdots, u(k)$. Here optimal means the minimal variance.

It is convenient to reformulate the ARX model to a state-space form. The ARX model can be reformulated in an innovation form as follows.

$$
\begin{aligned}
& {\left[\begin{array}{c}
\hat{x}_{1}(k+1) \\
\hat{x}_{2}(k+1) \\
\vdots \\
\hat{x}_{n}(k+1)
\end{array}\right]=\left[\begin{array}{ccccc}
-A_{1} & I & 0 & 0 \cdots & 0 \\
-A_{2} & 0 & I & \cdots & 0 \\
\vdots & \vdots & 0 & \ddots & 0 \\
-A_{n} & 0 & 0 & 0 & I
\end{array}\right]\left[\begin{array}{c}
\hat{x}_{1}(k) \\
\hat{x}_{2}(k) \\
\vdots \\
\hat{x}_{n}(k)
\end{array}\right]} \\
& +\left[\begin{array}{c}
B_{1} \\
B_{2} \\
\vdots \\
B_{n}
\end{array}\right] u(k)+\left[\begin{array}{c}
K_{1} \\
K_{2} \\
\vdots \\
K_{n}
\end{array}\right] \epsilon(k) \\
& y(k)=\left[\begin{array}{lllll}
I & 0 & 0 & \cdots & 0
\end{array}\right]\left[\begin{array}{c}
\hat{x}_{1}(k) \\
\hat{x}_{2}(k) \\
\vdots \\
\hat{x}_{n}(k)
\end{array}\right]+\epsilon(k)
\end{aligned}
$$

where $n=\max \left(n_{a}, n_{b}\right)$ and $K_{i}=-A_{i}, i \in\{1, \cdots, n\}$. The state, $\hat{x}(k) \in$ $\mathbb{R}^{p}$, appears through a realization process of the input-output model (1). The realization (2) can be found in, e.g. Goodwin and Sin (2013)[p. 263].

For notational simplicity, let us rewrite (2) as follows.

$$
\begin{aligned}
\hat{x}(k+1) & =\mathcal{A} \hat{x}(k)+\mathcal{B} u(k)+\mathcal{K} \epsilon(k) \\
y(k) & =\mathcal{C} \hat{x}(k)+\epsilon(k) .
\end{aligned}
$$




\subsubsection{Reasons for selecting the ARX model structure with the $L S$ algorithm}

We are particularly interested in the data obtained from systems operating in closed-loop, because interrupting existing systems for an accurate model have raised practical issues, e.g. comfort violation, and require human labor which may hurt the overall economics of the RTU coordination.

There are several advantages in using the simple ARX model structure. The LS can be easily generalized to a real-time identification, i.e. the recursive LS (RLS). This allows adaptation to the aforementioned time varying system properties, e.g. performance degradation of RTUs. Furthermore, Anderson and Johnson (1982); Goodwin and Sin (2013); Johnstone et al. (1982) showed that the RLS possesses global exponential stability for deterministic systems. This means the RLS ensures robustness with respect to an initial guess of the parameters and the numerical noises. Because the LS and the RLS are fundamentally the same, the LS is also numerically robust and computationally efficient. This is clearly beneficial to handle a large parameter estimation problem.

Despite the benefits, there are disadvantages in choosing the simple model structure. Assume the true system follows the linear system form; $y(k)=$ $G_{T}(z) u(k)+H_{T}(z) e(k)$, where $G_{T}(z), H_{T}(z)$ are rational polynomials in $z$ and $e(k)$ is a zero-mean white noise process. The $H_{T}(z)$ describes the output disturbance model for the unmeasured disturbances in buildings. From (1), it is clear that the ARX model structure assumes $H_{T}(z)$ as $\frac{1}{A\left(z^{-1}\right)}$. However the simple disturbance model is a poor representation of the complex disturbances in buildings. The discrepancy between $\frac{1}{A\left(z^{-1}\right)}$ and $H_{T}(z)$ causes the LS to be inconsistent especially when closed-loop I/O data ${ }^{2}$ is used for model estimation, since closed-loop operation causes correlation between the inputs and disturbances.

However, fortunately, this LS inconsistency is small for the RTU coordination problem. This is because; 1) our prediction horizon is relatively short, e.g. 30 min, and 2) the ON/OFF control sequence of the RTU control, which looks like a pseudo random binary signal (PRBS), decorrelates the outputs from the unmeasured disturbances.

Despite the favorable factors in this RTU coordination problem, there could be some model-mismatch, possibly from the linearly approximated model for the complex RTU controlled building systems. Furthermore there are unpredictable or unmodeled disturbances, e.g. sudden occupancy load injection to the buildings. To tackle the practical difficulties, the estimated model will be modified (See Section 3.2).

Another difficulty in using the ARX model structure is the determination of

\footnotetext{
${ }^{2}$ We also tried several different identification methods and model structures that contain more general disturbance model set, e.g. MIMO ARMAX with prediction error methods (PEM) and state space model structure with subspace identification methods (SIM). However the approaches also suffer from the inconsistency due to the mis-specified disturbance model especially for closed-loop identification. See discussions in (Ljung and McKelvey, 1996; MacGregor and Fogal, 1995; Van den Hof, 1998)
} 
the polynomial degrees, $\left(n_{a}, n_{b}\right)$, in (1). With several experimental data sets obtained from different buildings, a parametric study was performed and $\operatorname{ARX}(2,2)$ was selected (Kim et al., 2014). Prediction performance of the $\operatorname{ARX}(2,2)$ will be provided in later sections.

To increase the reliability of the estimated model, some additional work can be added, e.g. the independent perturbation of set-points to decorrelate the inputs and the unmeasured disturbances. However the other techniques are not included in this paper, since the simulation and experimental results show the overall approach from the simple modeling together with the modification method works well for the RTU coordination problem due to the relatively short prediction horizon and the nature of ON/OFF sequences.

\subsection{Model Augmentation}

The modification for the system-model mismatch and the unmodeled disturbances is based on the assumption that the unknowns vary relatively slowly. For example, we can assume that an unpredictable occupancy load in the building system is a piece-wise constant signal where the time interval for each constant is larger than the time constant of the system. The assumption is reasonably valid for most buildings, e.g. restaurants, shopping centers, office buildings.

We can adopt a well-known method, the internal model principle, from adaptive controls (Åström and Wittenmark, 2013) and process controls (Maeder et al., 2009; Muske and Badgwell, 2002; Pannocchia and Rawlings, 2003), to handle the problem. The method augments a system model by adding another disturbance model to compensate for the modeling error and unmodeled disturbances. In our case, it has the following form.

$$
\begin{aligned}
{\left[\begin{array}{c}
x(k+1) \\
p(k+1)
\end{array}\right] } & =\left[\begin{array}{cc}
\mathcal{A} & B_{d} \\
0 & I
\end{array}\right]\left[\begin{array}{l}
x(k) \\
p(k)
\end{array}\right]+\left[\begin{array}{c}
\mathcal{B} \\
0
\end{array}\right] u(k)+w(k) \\
y(k) & =\left[\begin{array}{ll}
\mathcal{C} & C_{d}
\end{array}\right]\left[\begin{array}{l}
x(k) \\
p(k)
\end{array}\right]+v(k),
\end{aligned}
$$

where $w$ and $v$ are white noise processes having covariance matrices of $Q, R$, respectively. The $\mathcal{A}, \mathcal{B}, \mathcal{C}$ are defined in (2) and (3). The unstable integrating modes, $p$, which are not controllable but observable, represent the constant unknowns. In general, the parameters of $\left(B_{d}, C_{d}\right)$ have to be adjusted depending on the system of interest.

With the assumption of the piece-wise constant disturbances, we chose a simple but widely used solution (Muske and Badgwell, 2002) that can be achieved by letting $B_{d}=0$ and $C_{d}=I$. Since the disturbance states, $p$, only affect $y$ and

not $x$, it is called constant output disturbance model. For a stable system, as in our building system, the constant output disturbance model removes steady state offset caused by the model mismatch and the unmodeled disturbances (Qin and Badgwell, 2003).

For notation simplicity,

$$
A_{a}:=\left[\begin{array}{cc}
\mathcal{A} & 0 \\
0 & I
\end{array}\right], B_{a}:=\left[\begin{array}{c}
\mathcal{B} \\
0
\end{array}\right], C_{a}:=\left[\begin{array}{ll}
\mathcal{C} & I
\end{array}\right], x_{a}:=\left[\begin{array}{c}
x \\
p
\end{array}\right] .
$$


If $(\mathcal{A}, \mathcal{C})$ is detectable $e^{3}$ then the augmented system $\left(A_{a}, C_{a}\right)$ is detectable since the unstable integrating modes $p$ are always 'recorded' in $y$. Furthermore since the $(\mathcal{A}, \mathcal{C})$ is observable ${ }^{4}$, the augmented system is automatically detectable. With the assumption of stabilizability of $\left(A_{a}, \sqrt{Q}\right)$, the detectabilty ensures that a steady Kalman filter exists and is stable (See Anderson and Moore (2012) for the proof). Then Kalman gain, denoted as $K_{a}$, for the augmented system, can be obtained by solving the algebraic Ricatti equation

$$
P=A_{a}^{T}\left(P-P C_{a}^{T}\left(R+C_{a} P C_{a}^{T}\right)^{-1} C_{a} P\right) A_{a}+Q
$$

and $K_{a}=A_{a} P C_{a}^{T}\left(R+C_{a} P C_{a}^{T}\right)^{-1}$.

Therefore our final model is

$$
\begin{aligned}
\hat{x}_{a}(k+1) & =A_{a} \hat{x}_{a}(k)+B_{a} u(k)+K_{a} e(k) \\
y(k) & =C_{a} \hat{x}_{a}(k)+e(k) .
\end{aligned}
$$

where $e(k)=y(k)-\hat{y}(k \mid k-1)$.

\subsection{Control Problem Formulation}

From this point, we restrict our discussion to the cooling case only for notation simplicity. One of our control objectives is to minimize HVAC energy consumption while meeting thermal comfort. Energy consumption during the $d$-step look ahead horizon at the current time step $k$ can be represented as $\sum_{j=0}^{d-1}\left(\sum_{i=1}^{m} P_{i}(k+j) u_{i}(k+j)\right) \times T_{s}$, where $T_{s}$ is the sampling time. $P_{i}(k+j) \in \mathbb{R}$, $u_{i}(k+j) \in\{0,1\}$ represent the power and the OFF/ON mode for $i^{\text {th }}$ RTU stage at $(k+j)^{t h}$ time step. $m$ is the number of total RTU stages.

The energy consumption can be expressed in terms of the ratios of individual power to the sum of the powers of all stages

$$
\sum_{j=0}^{d-1} P_{t o t}(k+j)\left(\sum_{i=1}^{m} \operatorname{Pr}_{i}(k+j) u_{i}(k+j)\right) \times T_{s}
$$

where $P_{t o t}=\sum_{i=1}^{m} P_{i}$ and $P r_{i}=\frac{P_{i}}{P_{t o t}}$. The power varies significantly with outdoor air temperature. Since our prediction horizon $d$ is relatively short, the change of outdoor air temperature within the look ahead horizon could be negligible. Therefore $P_{i}(k) \approx P_{i}(k+1) \approx \cdots \approx P_{i}(k+d-1)$. Then (9) becomes

$$
P_{\text {tot }}(k) \sum_{j=0}^{d-1}\left(\sum_{i=1}^{m} \operatorname{Pr}_{i}(k) u_{i}(k+j)\right) \times T_{s} .
$$

\footnotetext{
${ }^{3}$ We say the pair $(A, B)$ is stabilizable if $(A, B)$ has no uncontrollable and unstable hidden modes. Similarly, the pair $(A, C)$ is said detectable if $(A, C)$ has no unobservable and unstable hidden modes (Callier and Desoer, 1991).

${ }^{4}$ Note the ARX model (2) has an observer form.
} 
Our assumption is that the power ratios, $P r_{i}(k)$, are approximately the same as the ratios at the rated condition. To support this, consider a vapor compression cycle. When $T_{O A}$ becomes higher, more compressor power is needed in order to pump the refrigerant to the higher condenser pressure. The power increments differ from unit to unit, but the trend is the same for all units. Therefore the ratios are somewhat insensitive to $T_{O A}$. In other words, $\operatorname{Pr}_{i}(k)$ is relatively constant and can be approximated with the rated powers as long as the performance did not regrade. The assumption was employed in the case studies for simplicity.

Another control objective is reducing RTU compressor ON/OFF cycling. The inclusion of the short cycling reduction, i.e. the prevention of frequent compressor ON/OFF switching, is because the cycling reduces the life and deteriorates the performance of the unit (COP). When the compressor turns off, the refrigerant mitigates from the high pressure region, i.e. the condenser, to the low pressure region, i.e. the evaporator (Mitchell and Braun, 2013, pp 418419). The inverse heat flow reduces the time averaged cooling capacity of the unit. Furthermore when the stage turns back on, the compressor needs to work to recreate the low and high pressure regions associated with the evaporator and condenser. Therefore the cycling reduces the time averaged performance of the unit. To this end, we adopt a simple heuristic strategy: the mode of any RTU must be maintained during the $d$-step look ahead time horizon. Obviously when the horizon is small, it may cause significant cycling. On the other hand, choosing a large $d$ may result in thermal comfort constraint violations.

With the stated assumptions, the control problem formulation is:

$$
\min _{u \in\{0,1\}^{m}}\left(\sum_{i=1}^{m} P_{i} u_{i}\right) \times d \times T_{s}
$$

s.t.

$$
\begin{aligned}
& T_{l} \leq E\left(y(k+d) \mid \mathcal{G}_{k}\right) \leq T_{u} \\
& u_{i} \in\{0,1\}
\end{aligned}
$$

where $P_{i}$ is the rated power for $i^{t h} \mathrm{RTU}, E\left(y(k+d) \mid \mathcal{G}_{k}\right)$ is the optimal $d$-step prediction given in (13) and $\mathcal{G}_{k}=\{y(k-1), y(k-2), \cdots, u(k+d-1), u(k+$ $d), \cdots, u(k-1), u(k-2), \cdots\}$. Note that we only want to regulate the $d$-step ahead predicted temperatures within a temperature boundeomfort bound ${ }^{0}$ and not all of the predicted temperatures for less than the $d$ steps. This is because our prediction horizon, $d$, is relatively short, e.g. $30 \mathrm{~min}$ to 1 hour. The $d$ step temperature regulation reduces the large number of inequality constraints that would be necessary if all of the predicted temperatures were constrained. Furthermore due to the cycling-prevention strategy, the number of variables to be optimized has been reduced significantly. However, (11) is a binary integer programming problem, which could be computationally demanding especially for buildings equipped with a large number of RTUs. With this concern, we chose the linear programming relaxation of the binary integer program. In other words, 
$0 \leq u_{i} \leq 1$ is imposed instead of the integer constraints to yield an approximate solution and the solution is rounded in the end.

To derive $E\left(y(k+d) \mid \mathcal{G}_{k}\right)$, the following can be shown.

$$
\begin{aligned}
E\left(y(k+d) \mid \mathcal{G}_{k}\right)= & C_{a} A_{a}^{d} \hat{x}_{a}(k)+C_{a}\left(A_{a}{ }^{d-1} B_{a} u(k)\right. \\
& \left.+A_{a}{ }^{d-2} B_{a} u(k+1)+\cdots+B_{a} u(k+d-1)\right) .
\end{aligned}
$$

Since we imposed the constraint $u(k)=u(k+j), \forall j \geq 0$, the above (12) yields

$$
\begin{aligned}
E\left(y(k+d) \mid \mathcal{G}_{k}\right)= & C_{a} A_{a}{ }^{d} \hat{x}_{a}(k) \\
& +C_{a}\left(A_{a}{ }^{d-1}+A_{a}{ }^{d-2}+\cdots+I\right) B_{a} u(k) .
\end{aligned}
$$

This formula provides an explicit way to express the inequality condition, $T_{l} \leq E\left(y(k+d) \mid \mathcal{G}_{k}\right) \leq T_{u}$ in (11).

\subsection{Input update frequency}

When the prediction horizon, $d$, is fixed, how frequently the manipulated input should be updated, say input update frequency, needs to be discussed. If the input is updated every time step, as in a conventional MPC, it could cause significant compressor cycling especially when the RTUs have similar performances. If the input is updated every $d$ steps, the disturbance rejection of the controller may become poor. Therefore the control input update frequency is one of the most important control parameters determining the controller performance.

We propose an adaptive selection scheme for the input updating time. Suppose a control decision was made at $k$ and the input $u(k)$ has been applied from $k$ to $k+j-1$ where $1 \leq j \leq d$. As $y(k+j)$ is available, the $j$-step ahead prediction error, $y(k+j)-E\left(y(k+j) \mid \mathcal{G}_{k}\right)$, can be calculated. The algorithm to select the updating time consists of: 1 ) if the prediction error is larger than a threshold, then update the input. 2) Otherwise, $u(k)$ is applied at $k+j$. The process is repeated for all $j$ until the input is updated. The algorithm is based on the following intuition; make a new decision if unexpected behavior occurs.

\subsection{Summary of Supervisory Controller and Further Discussions}

A basic control structure is shown in Fig. 1 which has a standard form of the model based controller ${ }^{5}$. The parameter estimator was explained in Section 3.1 and $(8)$ is the state observer in this figure. The observer provides an estimated state, i.e. $\hat{x}_{a}$ in (13), and allows the optimizer to solve the control objective (11).

The overall procedure follows well-developed methods from system identification and process controls in order to make a reliable RTU coordinator for small/medium commercial buildings.

\footnotetext{
${ }^{5}$ The disturbances in Fig. 1 represent any uncontrollable sources which interact with the building system, e.g. weather or occupancy gains.
} 


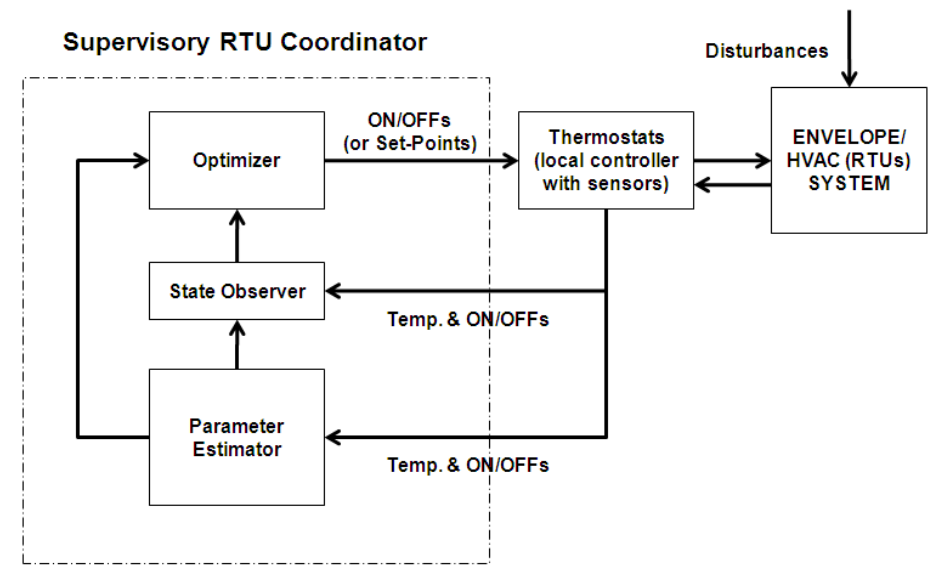

Figure 1: Basic structure of supervisory RTU coordinator

Note that this approach only requires thermostat temperatures and ON/OFF signals as measurements and does not require weather or other data for implementation. Because of the minimal sensor requirement and self-learning inputoutput response modeling, the controller is termed PnP (Plug-and-Play). The control objective is to minimize HVAC energy consumption while meeting comfort constraints and reducing compressor cycling and possibly reducing peak demand.

The use of only thermostat outputs as input measurements for the coordination algorithm is an important feature that enables a scalable and low-cost implementation. However due to the lack of other disturbance information, only a short prediction horizon, say less than an hour, is feasible. The short prediction horizon restricts an active usage of the building's thermal mass and it is a significant weakness of the controller. One possible enhancement to improve longer term predictions would be to add an outdoor air sensor which is relatively inexpensive. In addition, rated RTU powers are used in the coordination algorithm to keep implementation costs low. However the assumption could be poor if RTU performance were degraded due to the presence of faults (e.g., fouled condensers) or poor installation. In this case, some measurement of actual RTU power would lead to a better solution for optimal coordination of the RTUs.

\section{Demonstration of PnP RTU coordinator}

For demonstrations of the PnP, two case-study buildings, a restaurant (Case 1) and a gymnasium (Case 2), were considered. In Case 1, the MIMO controller modeling approach was validated with experimental data, while the closed-loop controller performance was evaluated in a simulation platform. In Case 2, both modeling and controller performance were tested experimentally. 


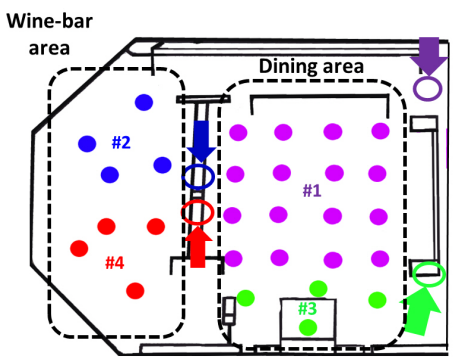

(a) Locations of thermostats and supply air diffusers

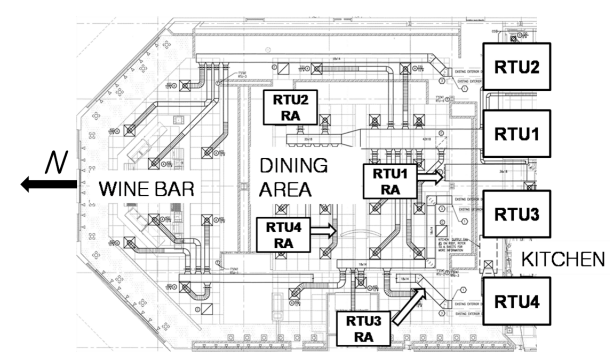

(b) Locations of return air vents

Figure 2: Characteristics of restaurant case study building: The filled circles and empty circles represent the locations of supply air diffusers and current thermostats. The different colors correspond to the different numbered RTUs $(\#)$

A restaurant in suburban Philadelphia is a test site for the Department of Energy through the Consortium for Building Energy Innovation (CBEI) (see Fig. 2a). The indoor restaurant area is about $70 \mathrm{ft}(12.86 \mathrm{~m})$ long, $65 \mathrm{ft}(19.81$ $\mathrm{m}$ ) wide and $11 \mathrm{ft}(3.35 \mathrm{~m})$ high. After analyzing the restaurant using available data, some important characteristics were found and are summarized as follows.

- Four RTUs serve two different areas in the restaurant (main dining and wine bar in Fig. 2a). RTU1 and RTU3 serve the dining area and RTU2 and RTU4 serve the wine bar.

- RTU1 is a two-stage unit, while the others are identical and single-stage. The overall COP (Coefficient of Performance) of the two-stage RTU (RTU1) is around $35 \%$ higher than the others and its COP for sensible cooling is around $50 \%$ higher. It also has about 3 times the cooling capacity.

- There are no return air vents in the wine bar area where the supply air diffusers of RTU2 and RTU4 are located. All return vents are located in the dining area. Due to the air duct system, there is a significant net flow from the wine bar to the dining area (see Fig. 2b). This prevents effective cooling of the wine bar area using the more efficient RTU1.

- Since some supply air diffusers of RTU3 are located near the return air vent of RTU4 and some supply diffusers of RTU1 are located near the RTU3 return air vent (see Fig. 2b), there can be significant interaction between the RTU1 and 3.

- The thermostats for RTU2 and RTU4, blue and red arrowed circles in Fig. 2a, are close to supply air diffusers for RTU1 (purple circles) and are likely affected by RTU1. ${ }^{2}$ 
Furthermore, all thermostats, marked as circles in Fig. 2a, are located relatively close to the supply air diffusers of RTU1. Based on the above analysis, a near optimal coordinator would utilize RTU1 as much as possible and minimize the use of the other RTUs to save energy. We will analyze our controller in Section 4.1.4 based on this observation.

\subsubsection{Virtual control test bed}

Although the controller modeling approach described in Section 3 was validated with experimental data, the PnP performance was evaluated in a simulation model. Fully-mixed assumption-based building energy simulation tools, e.g. EnergyPlus (Crawley et al., 2000) and TRNSYS (Klein et al., 2004), have limited applicability for evaluating control algorithms for RTU coordination because the spatial differences of zone air temperatures cannot be captured. Therefore, we developed a simulation tool that can assess both energy and comfort performance for these types of applications so that the benefits of RTU coordination and control can be evaluated. The simulation tool for the restaurant includes a reduced-order transient model for the indoor environment that was generated from CFD results, a detailed transient model for the building envelope (walls, roof, and floor) generated from a physical description, and equipment and air distribution models based on manufacturers' information and measurements that capture the effects of cycling. The integrated model was validated using measurements from the field site. See Kim et al. (2015) for a detailed description and validation of the model.

\subsubsection{Demonstration of low cost predictor modeling approach}

Temperature sensors corresponding to the thermostats were located as shown in Fig. 2a. The temperatures and each RTU ON/OFF mode were recorded with a five-minute sampling time where each mode was controlled by a thermostat (The thermostat used conventional staged control with deadbands of $0.5^{\circ} \mathrm{C}^{2}$. feedback control: if measured temperature is higher than user defined set-points, then turn on the RTU and vice versa ${ }^{2}$ ). The data sets were obtained from the conventional closed-loop system.

The measured temperature and $\mathrm{ON} / \mathrm{OFF}$ mode data were recorded from the site. A sample I/O data set for conventional thermostat control, August 22, 2013 to September 5, 2013, is shown in Fig. 3. Using the I/O data, the identification algorithm described in Section 3.1 was implemented with a 7 -day data period with the $\operatorname{ARX}(2,2)$ model structure as discussed in Section 3.1.1. After training, the output disturbance model was augmented as described in Section 3.2. In order to validate the modeling approach, the $d$-step predicted outputs calculated by (12) were compared with the measured outputs for the other data set. The results when $d=12$ are shown in Fig. 4. Since the sampling time is five minutes, it is equivalent to a one-hour look ahead prediction. The results match very well for the four thermostat temperatures despite significant spatial variations of temperature between the zones (around $5{ }^{\circ} \mathrm{C}$ in the worst case between thermostats 1 and 4 ). Sample standard deviations for the one-hour prediction errors are $0.69,0.29,0.56$ and $0.40\left({ }^{\circ} \mathrm{C}\right)$, respectively. 


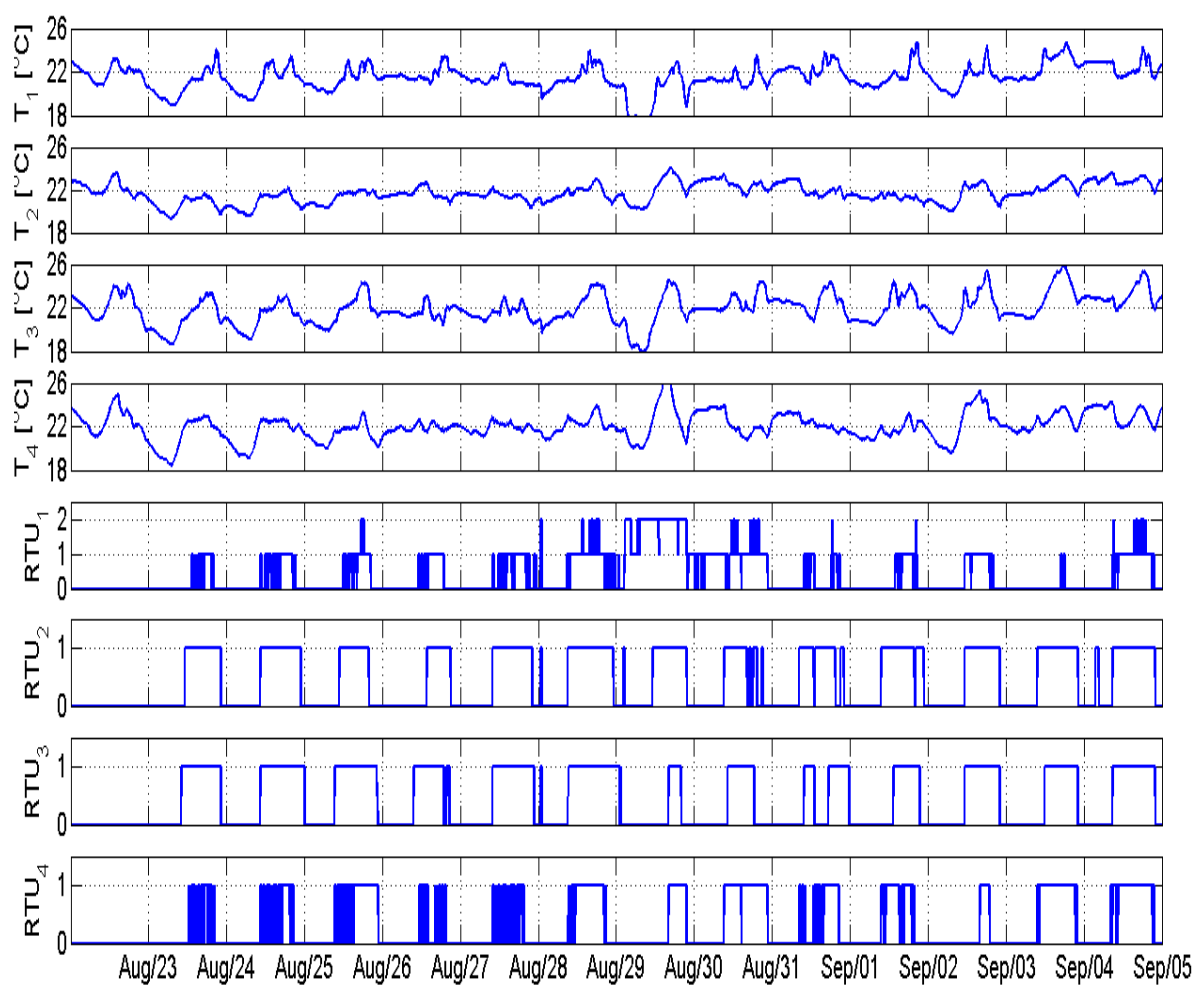

Figure 3: Sample thermostat and RTU stage measurements for the restaurant

In order to better understand the reliability of the estimated model, the accuracy of the estimated parameters was checked. The trace of the covariance matrix of the parameter errors, denoted as $\operatorname{tr}\left(E\left(\tilde{\theta}_{N} \tilde{\theta}_{N}^{T}\right)\right)$ where $\tilde{\theta}_{N}=\hat{\theta}_{N}-\theta_{T}$, is used for the measure of accuracy ${ }^{6}$. In this study, three different models were obtained from three different data sets with the same model structure and training algorithm. Each data set had 2105 data points which corresponds to one week of data for the 5-minute sampling time. The comparisons are shown in Table 1. For all cases, the LS algorithm with the $\operatorname{ARX}(2,2)$ model structure provided accurate models.

\subsubsection{Simulation test for PnP Controller}

To evaluate the controller performance for the restaurant case study, the setpoints for the conventional controls were set as $22{ }^{\circ} \mathrm{C}$ during an occupied period for all zones and the specified temperature bounds that are shown in greyeomfort bounds ${ }^{0}$ for the PnP control were $\left[\begin{array}{ll}19 & 22\end{array}\right]^{\circ} \mathrm{C}$ for the period. For the unoccupied period, the set-points are $24{ }^{\circ} \mathrm{C}$ for the conventional control and the bounds

${ }^{6}$ For the estimation details of the covariance matrix, see (Ljung, 1999, p 284 and p 553). 


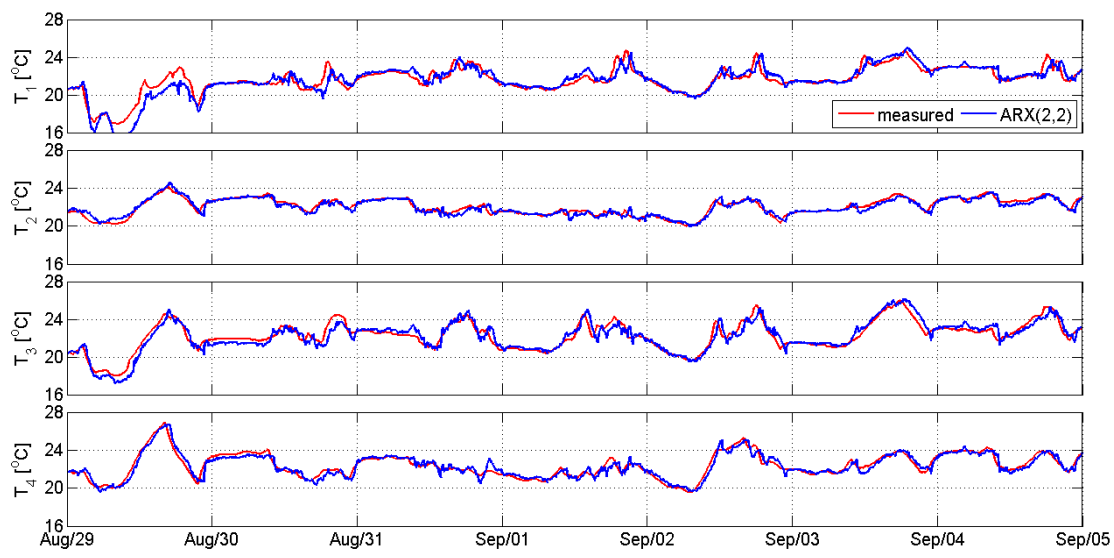

Figure 4: Model evaluation for the restaurant comparing one-hour predicted values to measured data

Table 1: The trace of the covariance matrix of LS estimation for three different restaurant data sets

\begin{tabular}{|c|c|c|c|}
\hline & data set 1 & data set 2 & data set 3 \\
\hline $\operatorname{tr} E\left(\tilde{\theta}_{N} \tilde{\theta}_{N}^{T}\right)$ & 0.0250 & 0.0242 & 0.0039 \\
\hline
\end{tabular}

were $\left[\begin{array}{ll}19 & 24\end{array}\right]^{\circ} \mathrm{C}$. The virtual testbed mentioned in Section 4.1.2 was executed in the Matlab Simulink environment with a stiff ordinary differential equation numerical solver, ode15s, that provides variable simulation time steps. A onemonth simulation (July) was performed for each controller. TMY3 weather data was used for the simulation.

Since the virtual testbed was validated with measurements, it made sense to utilize it for an initial evaluation of the controller. For consistency, the PnP controller MIMO model was trained using outputs from the simulation rather than the experiments. Based on the discussion in Section 4.1.3, the $\operatorname{ARX}(2,2)$ model structure was selected. One-week historical I/O data from the simulation results under the conventional control was utilized for populating the model. A 5-minute sampling time was chosen for the conventional and the PnP controllers. In this case study, a 30-min look ahead horizon was selected that corresponds to $d=6$. The prediction error threshold for updating the control horizon discussed in Section 3.4 was set to $0.5^{\circ} \mathrm{C}$.

Fig. 5a-6b show sample comparisons between the conventional and PnP control from the one-month (July) simulation results. Recall that RTU1 is a twostage unit while the others are single-stage units (see Section 4.1.1). Output stages of 1 and 2 of RTU1 in Fig. 6a - 6b indicate the first and second stages of RTU1, respectively. The OFF state is denoted as 0 in the figures. 
Fig. 5a and 5b show the temperature profiles under conventional feedback and $\mathrm{PnP}$ control, respectively. Temperatures for the $\mathrm{PnP}$ are more oscillatory than the conventional but are mostly within the temperature boundseomfort bounds ${ }^{0}$ (recall that the control objective is not set-point tracking). For a few times, there are comfort violations of the PnP, e.g. around 12:00 PM for the first day in Fig. 5b. This is because: 1) linear programming relaxation was employed to approximate a solution, 2) there could be modeling error and 3) there is prediction error due to the unknown disturbances. However the violations are comparable to the conventional case in Fig. 5a.

A remark can be made on the thermostat temperatures: the temperatures for the $\mathrm{PnP}$ are around $1-2{ }^{\circ} \mathrm{C}$ lower than the temperatures for the conventional controller for the occupied time. However this does not imply the PnP uses more HVAC power, because one can meet higher cooling load with lower HVAC power consumption if more efficient equipment is operating more of the time. Note the increased usage of the second stage unit of RTU1 for the PnP in Fig. 6b compared to the conventional control in Fig. 6a. As mentioned in Section 4.1.1, RTU1 is much more efficient than the others.

The run time fraction (RTF) is defined as the fraction of ON period to the total period. The RTF for each RTU for the one-month period is summarized in Table 2. $R T U_{1,1}$ and $R T U_{1,2}$ denote the first and second stages of $R T U 1$, respectively. The metric provides information on how each RTU is utilized. For the conventional control, note the low RTF of RTU1 stage 2, and the high RTF of the inefficient RTUs, i.e. RTU2-4. This is because it does not consider the effectiveness of these RTUs. On the other hand, the PnP controller exhibits the opposite behavior. Fig. $5 \mathrm{~b}$ shows that the temperatures $T_{2}-T_{4}$ can be maintained without utilizing the inefficient RTUs which can deliver cold air directly to the thermostats.

Based on the results above, it is clear that the $\mathrm{PnP}$ attempts to adjust all thermostat temperatures with only one RTU, if possible, and it is obviously preferable behavior as discussed in Section 4.1.1. Since RTU1 has three times higher capacity than the other units (see Section 4.1.1), then its increased usage with cycling of its stages leads to some increased oscillation in the thermostat temperatures. However, again, it is mostly within the temperature boundseomfort bounds ${ }^{0}$. Furthermore the PnP does not raise the temperatures uniformly to the comfort upper bound, i.e. $22^{\circ} \mathrm{C}$. This is because if the PnP turns off the second stage of RTU1, then $T_{2}$ and $T_{4}$, which are already close to the upper bound as shown in Fig. 5b, most likely violate the constraint. The other option to keep $T_{2}$ and $T_{4}$ below the upper comfort constraint would be to turn on the less efficient RTU2 and/or RTU4. However, this would lead to greater energy consumption.

Table 3 shows daily averaged HVAC energy consumption for fans and compressors. The PnP control achieved $20.69 \%$ savings for this test period compared to the conventional control, with the savings coming from utilization of the more efficient RTU. The significant peak demand reduction, about $30 \%$ compared to the conventional, is intriguing, since the controller objective (11) does not explicitly consider the peak demand reduction. The explanation on 

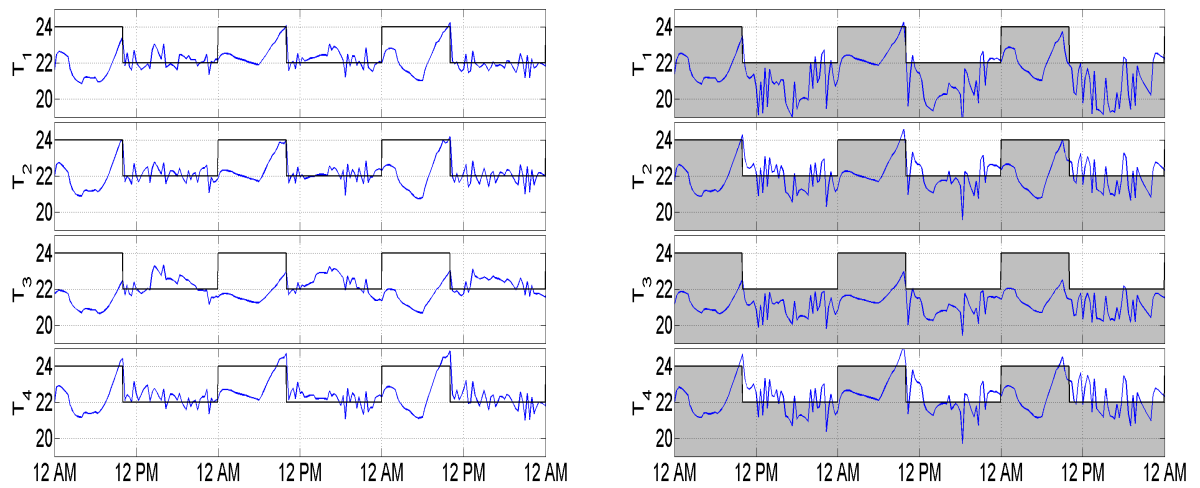

(a) under conventional feedback control, heating(b) under plug-and-play control, grey: temperais deactivated ture bounds

Figure 5: Temperature profiles (simulation) for restaurant case study; unit: ${ }^{\circ} \mathrm{C}$

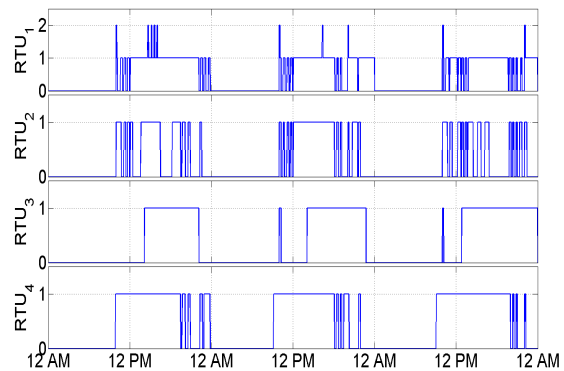

(a) under conventional feedback control

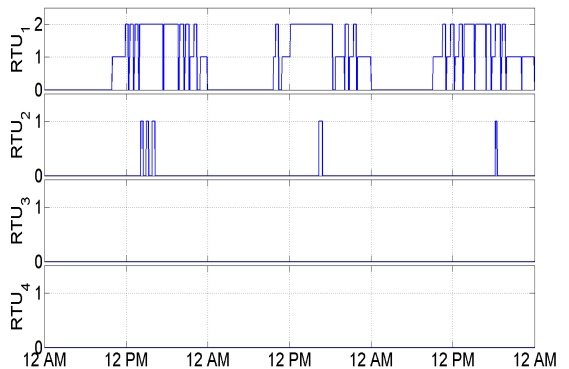

(b) under plug-and-play control

Figure 6: RTU stages (simulation) for restaurant case study

the mechanism of the demand savings will be provided in Section 4.2.1.

\subsubsection{Impact of thermostat locations}

The $\mathrm{PnP}$ was designed to regulate measurable temperature as shown in the control objective (11). Therefore it is natural to check thermostat temperatures for evaluating performance. However it is important to emphasize that the temperatures may not be the representative of zone air temperatures due to poor thermostat locations. As previously mentioned, thermostats 2 and 4 are located near the supply air diffusers of RTU1 and are therefore mostly influenced by RTU1. Furthermore, there is a net air flow from the wine bar area to the dining area due to the return duct system layout, which prevents efficient cooling of the wine bar area by RTU1, as discussed in Section 4.1.1. Therefore the regulation of the temperatures at thermostats 2 and 4 in Fig. $6 \mathrm{~b}$ does not imply the regulation of the wine bar zone. Fig. 7a shows volume-averaged 
Table 2: Comparisons of run time fraction (RTF) of each RTU for restaurant case study (one month simluation period).

\begin{tabular}{|c||c|c|c|c|c|}
\hline & $R T U_{1,1}$ & $R T U_{1,2}$ & $R T U_{2}$ & $R T U_{3}$ & $R T U_{4}$ \\
\hline Conventional & 0.45 & 0.02 & 0.25 & 0.45 & 0.57 \\
\hline Plug-and-Play & 0.57 & 0.26 & 0.03 & 0.02 & 0.00 \\
\hline
\end{tabular}

Table 3: Comparisons of HVAC energy consumption for restaurant case study

\begin{tabular}{|c||c|c|}
\hline & Conventional & Plug-and-Play \\
\hline Energy consumption (kW-h/day) & 284.49 & 225.64 \\
\hline Relative energy savings [\%] & - & 20.69 \\
\hline Peak demand (kW) & 29.08 & 20.15 \\
\hline Relative demand savings [\%] & - & 30.71 \\
\hline
\end{tabular}

temperatures for the two zones ${ }^{7}$. The temperatures are around $1-2^{\circ} \mathrm{C}$ above the comfort upper bound, marked as black solid lines, for both the conventional and PnP. In particular, because of the low utilization of RTUs 2 and 4 for the PnP, the wine bar zone air temperature reaches up to $3^{\circ} \mathrm{C}$ above the temperature boundseomfort bound ${ }^{08}$. Therefore it is important to evaluate the performance of the PnP after retrofitting thermostat locations.

We moved the thermostat locations for all four RTUs such that the temperatures are more representative of the volume-average temperatures for the areas served by those RTUs. For detailed information about the sensor retrofit, refer to Kim et al. (2015). After the model estimation process with a new data set generated from the retrofitted system, simulations were implemented for the conventional and the $\mathrm{PnP}$ under the same simulation setup.

Comparisons of RTU run-time fractions (RTF) are shown in Table 4.

Table 4: Comparisons of run time fraction (RTF) of each RTU for restaurant case study after retroffiting thermostat locations (one month simluation period).

\begin{tabular}{|c||c|c|c|c|c|}
\hline & $R T U_{1,1}$ & $R T U_{1,2}$ & $R T U_{2}$ & $R T U_{3}$ & $R T U_{4}$ \\
\hline Conventional & 0.53 & 0.07 & 0.61 & 0.61 & 0.29 \\
\hline Plug-and-Play & 0.55 & 0.21 & 0.61 & 0.07 & 0.27 \\
\hline
\end{tabular}

For the PnP, the RTFs of the less efficient RTUs increased significantly compared to Table 2. This result indicates that RTU1 cannot be used to regulate

\footnotetext{
${ }^{7}$ We used volume-averaged temperatures for a comfort metric. For the calculations, please refer to Kim et al. (2015).

${ }^{8}$ Note that comfort problems occurred because of the poor thermostat locations and the controller is only designed to maintain temperatures that are measured by the thermostats.
} 


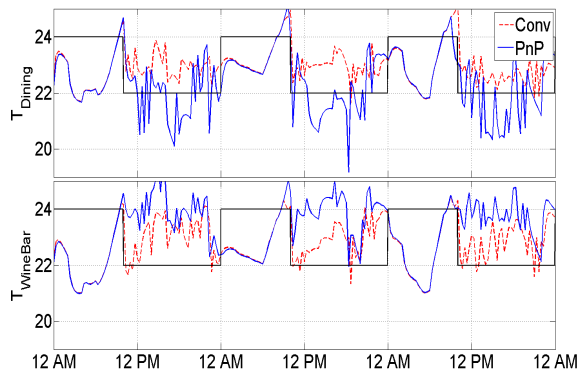

(a) Before a sensor retrofit

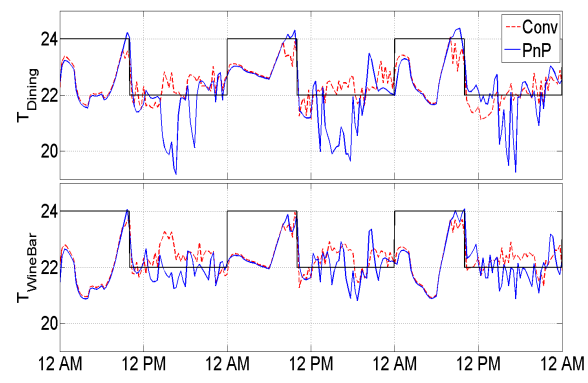

(b) After a sensor retrofit

Figure 7: Comparisons of volume-averaged zone air temperatures for wine bar $T_{\text {WineBar }}$ and dining areas $T_{\text {Dining. }}$ RTU2 and 4 serves the wine bar area while RTU1 and 3 serves dining area; unit: ${ }^{\circ} C$

temperatures within the wine bar zone because the net air flow is from the wine bar to the dining area. However, the $\mathrm{PnP}$ still finds a way to save energy by coordinating RTU1 and RTU3. The RTF of RTU3 is 0.07 , which is considerably smaller than the conventional case, which has a value of 0.61 . This confirms that the PnP algorithm learns the system characteristics. Due to the higher utilization of inefficient RTUs, the energy savings decrease from $20 \%$ to $10 \%$ due to the change of thermostat locations. However the volume-averaged temperatures are regulated for both controllers as shown in Fig. 7b; the comfort violations are less than $1^{\circ} \mathrm{C}$ for both controllers. ${ }^{2}$

\subsection{Case 2: Gymnasium, Experimental Demonstration of the PnP Controller}

The proposed algorithm was demonstrated at a test site, a gymnasium, in Knoxville, TN, U.S. The gym is a research field site for Oak Ridge National Laboratory. The system characteristics are summarized in Section 4.2.1 and comparisons between conventional and PnP control are provided in Section 4.2.4. Although the controller was implemented for several weeks, only one week of data is presented in the comparisons.

\subsubsection{System description and peak demand reduction strategy}

The test site is shown in Fig. 8a and has the following characteristics:

- 4 RTUs (identical 10-ton units) serve the gymnasium with two-stage compressors.

- There is strong coupling between the four zones served by the RTUs because of the supply air diffuser system and thermostat locations.

The identical RTUs implies that there may not be significant energy savings potential associated with RTU coordination based on optimizing for utilizing the 


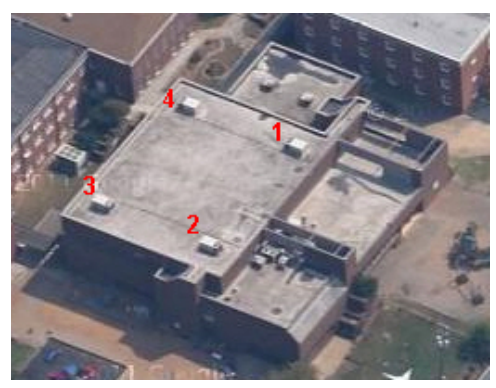

(a) External view of the gym

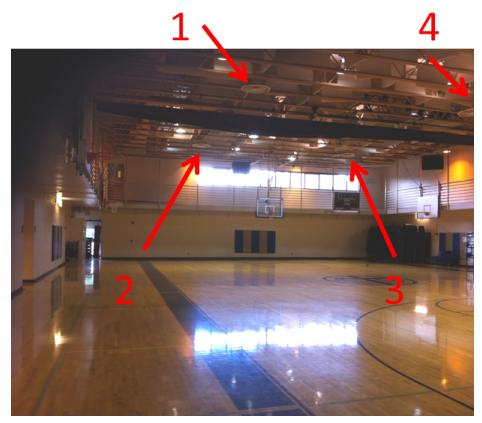

(b) Internal view of the gym (taken from \#1)

Figure 8: PnP demonstration site, a gymnasium, \#1-4 represent the RTU numbering used in this paper.

most efficient RTU. However, the $\mathrm{PnP}$ controller can also significantly reduce peak power demand compared to a conventional feedback control.

Consider a zone served by several 2-stage RTUs. Suppose a temperature set-point is fixed and a constant cooling load is injected to the zone served by one of the RTUs. Depending on the load, the previous time history of control, and the operation of other RTUs, a conventional control may lead to operation of either $1^{\text {st }}$ or $2^{\text {nd }}$ stage of cooling for an individual RTU as depicted in Fig. 9. Second stage cooling would result in a large cooling rate and peak power but shorter operating period, whereas first stage would operate longer with a lower peak power. Conventional feedback control could lead to RTU cycling patterns that emphasize shorter operation with higher peak power consumption than optimal from a demand perspective.

However the $\mathrm{PnP}$ algorithm predicts $d$-steps ahead and determines the least power RTU stage that can bring the zone temperature within the temperature boundsemfort bound ${ }^{0}$ for the $d$-step ahead prediction period (see Section 3.3). Note that the algorithm is equivalent to providing an appropriate cooling rate to meet the predicted load. For this example as in Fig. 9, the PnP would chose $1^{\text {st }}$ stage if it could keep the zone temperature within the comfort band for the specified prediction period (e.g., 1 hour). When $2^{\text {nd }}$ stage is considered in the $\mathrm{PnP}$, it would expect that the zone air temperature at the prediction point could be over-cooled while requiring more energy. This is because the $2^{\text {nd }}$ stage has to be maintained during the prediction period due to the short cycling preventing strategy (See Section 3.3). Therefore the PnP would not provide an excessive cooling capacity at a time under a part-load condition while a conventional control may do so even for a very low part-load condition.

As a result, the PnP reduces the peak demand compared to the conventional as in Fig. 9. Of course, when the cooling load requires RTU full capacity or when a building is served by a single stage RTU, there would be no opportunity for 


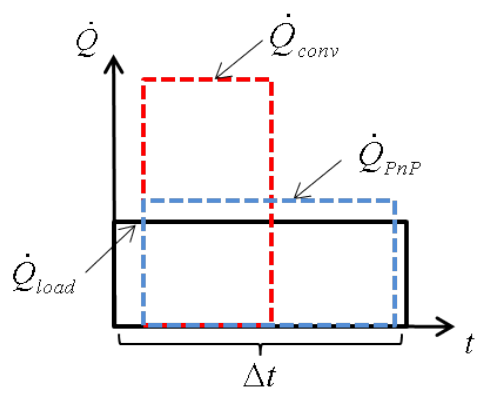

Figure 9: Conceptual demand reduction strategy for RTU cordination

demand reduction. However in practice, RTUs are selected to meet a peak load which may occur for a few days per a year, thereby RTUs work under a partload condition for most times. This means there is significant peak demand reduction potential almost always except for the peak-load days.

\subsubsection{Demonstration of low cost predictor modeling approach}

The October 2013 data shown in Fig. 10 was obtained from the test site. The sampling time was $5 \mathrm{~min}$ and the LS was performed with the $\operatorname{ARX}(2,2)$ model structure. 6 days data was used for model development and the model was validated with other data sets. One-hour predictions, i.e. 12-step ahead predictions, were compared to the thermostat temperature measurements in Fig. 11. The sample standard deviations of the one-hour prediction errors are $0.21,0.18,0.23,0.15{ }^{\circ} \mathrm{C}$ and the estimated the trace of the covaraince matrix of the parameters, i.e. $\operatorname{tr}\left(E\left(\tilde{\theta}_{N} \tilde{\theta}_{N}^{T}\right)\right)$, is 0.0476 .

\subsubsection{Control implementation}

The gym deployment site utilized thermostats having MODBUS access to key thermostat variables and control such as remotely selecting relay states. The configuration is shown in Fig. 12. In addition to the MODBUS network, the site also included sub metering data collection for each RTU. Some integration development and testing was required to obtain the desired communications functionality between a computer running the software (coded in Matlab R2010a) and the MODBUS thermostats. After that, additional functional testing of the algorithm was performed in order to verify safe operation. For a commercial implementation where thermostat communication is already available, the additional costs for implementation of the PnP control would be minimal once the infrastructure is in place for model training and algorithm execution.

\subsubsection{Comparisons of PnP to a conventional control}

Because weather and internal gains keep changing, fair performance comparisons between the two control algorithms are difficult in practice. In order to compare the PnP performance to the conventional control, the algorithms were switched daily. In other words, $\mathrm{PnP}$ was implemented for one day and the 


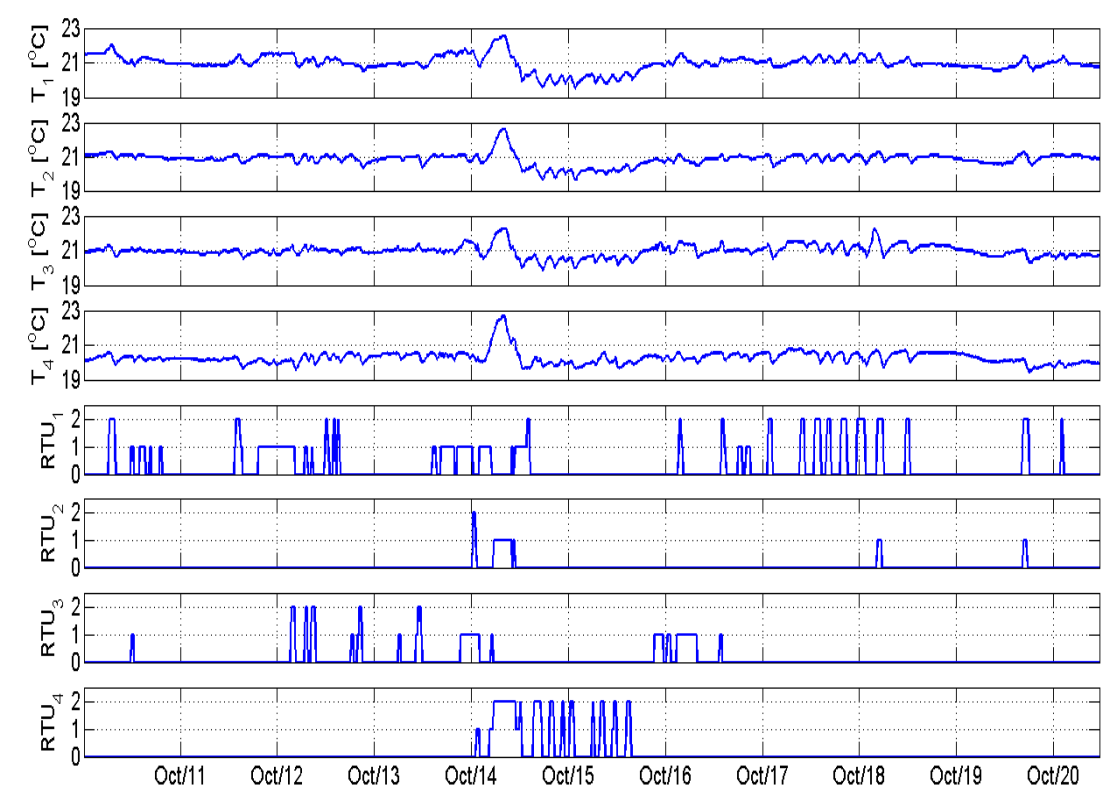

Figure 10: Sample thermostat and RTU stage measurements for the gymnasium

conventional control was implemented for the following day. In our demonstration, the $\mathrm{PnP}$ was run for even days, e.g. Sep/8 and Sep/10, and conventional control for odd days, e.g. Sep/7 and Sep/9. The switching was repeated for one week.

Sample results for conventional and $\mathrm{PnP}$ control are shown in Fig. 13a and 13b. The black line is the trajectory of thermostat temperature set-points and were adjusted by the occupants. Although the temperature set-points in the Figures are slightly different for a portion of the time, $0.28{ }^{\circ} \mathrm{C}\left(0.5^{\circ} \mathrm{F}\right)$, the differences are very small and are fair for the overall comparison period. For the $\mathrm{PnP}$, the set-point was used for the high temperature boundseomfort bound ${ }^{0}$. The PnP predicted one-hour ahead and the sampling time was 5 min. For the conventional control, 5-min sampling time was also used.

Fig. 13a and 13b show the $2^{\text {nd }}$ RTU was turned off for most of the time during the test periods for both control methods while the corresponding thermostat temperature measurements (green lines) are the lowest. These experimental results demonstrate there is significant interaction between RTUs for this typical open space buildings. ${ }^{2}$

In Fig. 13a, the zonal temperature fluctuations of the conventional control algorithm, denoted as Conv, are noticeable compared to PnP (shown in Fig. 13a). The zone temperature amplitude of Conv is around $1.7{ }^{\circ} \mathrm{C}\left(3^{\circ} \mathrm{F}\right)$ while it is around $0.8^{\circ} \mathrm{C}\left(1.5^{\circ} \mathrm{F}\right)$ for PnP. This is due to the inability of the conventional control to look ahead as described in Section 4.2.1, which leads to greater cy- 


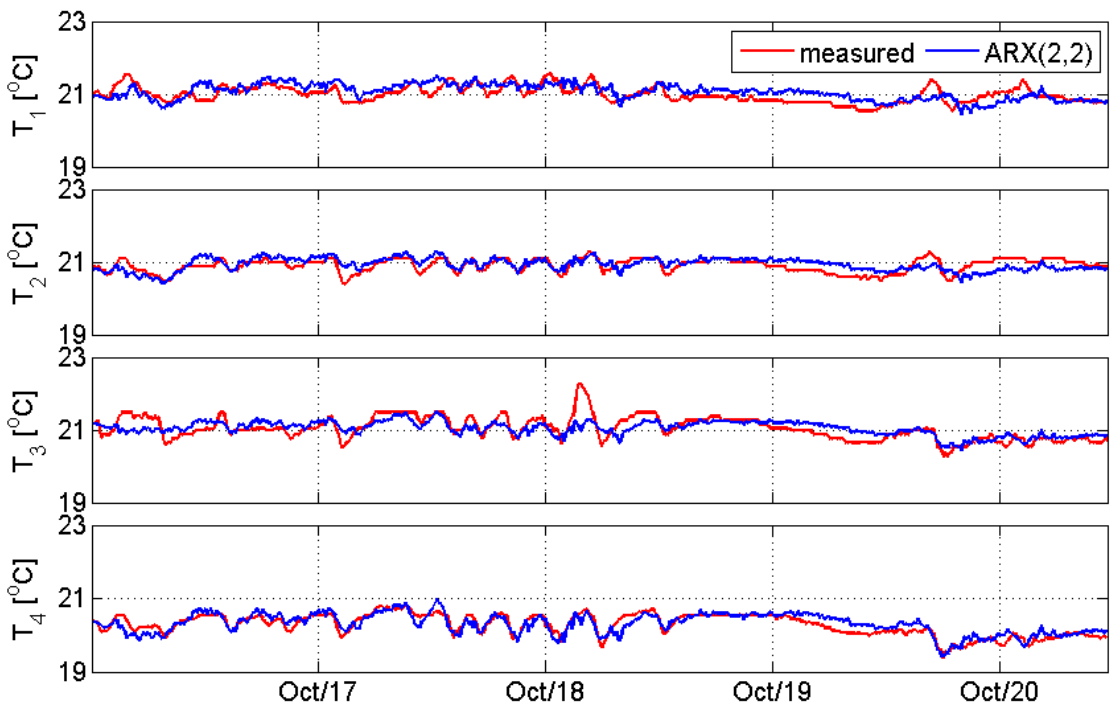

Figure 11: Model evaluation by comparing one-hour predicted values to measured thermostat data for gym case study

cling of units than is needed. For instance, at around 9:00 AM in Fig. 13a, energy gains cause a temperature rise. After a short period of time, three of the thermostat temperatures become higher than the $2^{\text {nd }}$ stage dead band, which is $0.33{ }^{o} C\left(0.6{ }^{o} F\right)^{9}$. Therefore, the conventional control leads all three of the associated RTUs to operate in $2^{\text {nd }}$ stage. At this point, 6 compressors were $\mathrm{ON}$ at the same time. The aggressive control action was maintained for about 20 minutes that led to the temperatures dropping rapidly and up to $1.11^{\circ} \mathrm{C}\left(2^{\circ} \mathrm{F}\right)$ below the set-point. The actual air temperatures were probably even lower due to the time constants of the thermostats. It is important to note that the aggressive cooling associated with the conventional control causes a significant electric demand even for these part load conditions.

On the other hand, as shown in Fig. 13b, PnP maintains control decisions for longer periods rather rapidly changing between $\mathrm{ON}$ and $\mathrm{OFF}$, but maintains the set-points better than conventional control. Notice that the PnP meets cooling loads with a maximum of only 2 compressors operating at any time for the whole day, while Conv uses up to 6 compressors. This is interesting because the activity schedules of the gym were more or less regular and the outdoor air temperatures for the sample days were not significantly different. Similar behaviors were confirmed for the all comparison days. The results imply that

\footnotetext{
${ }^{9}$ The small dead band is due to the high volume of the gym that is around $929 \mathrm{~m}^{2}(10,000$ $\left.f t^{2}\right) \times 7 m(22 f t)$. See Fig. 10 for the dynamics of the zone air)
} 


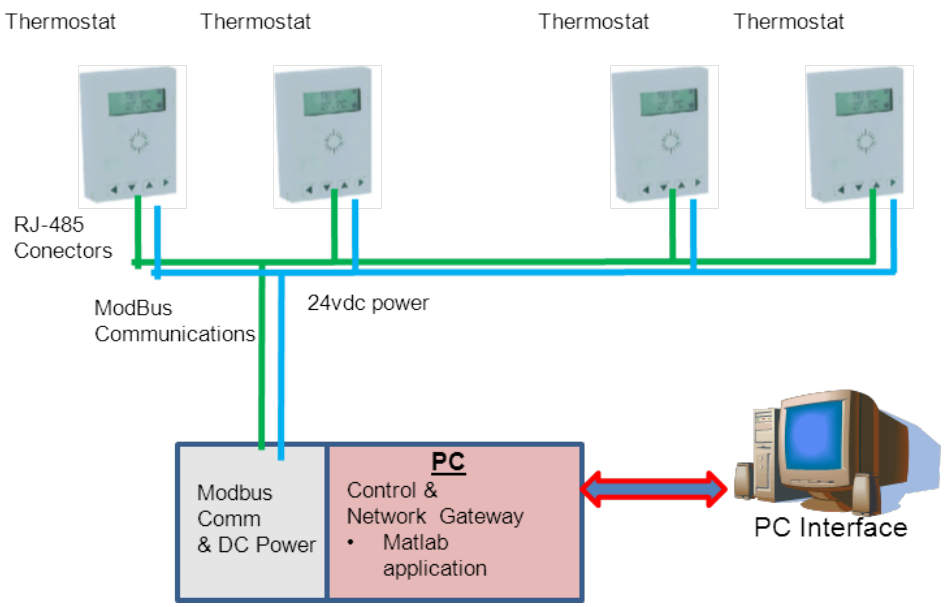

Figure 12: Deployment architecture for gym case study

there is significant demand reduction potential associated with the PnP control. It is also important to note that the model is based on data for RTU status and thermostat temperatures. As a result, the data-driven model accounts for the dynamics of thermostats.

Notice that PnP utilizes RTU3 primarily while rarely using RTU2 and RTU4 in Fig. 13b. The result is also intriguing since all RTUs are identical units. It turns out RTU3 or RTU1 can affect all of the thermostats because of the nature of the supply air distribution system, thermostat locations and geometry of the gym. Furthermore, the models automatically capture these system characteristics. These arguments are supported by results shown in Fig. 13b, since thermostat 2 and thermostat 4 temperatures are well regulated even though RTU2 and RTU4 are OFF for these days.

The performance comparisons for 4 consecutive days are shown in Fig. 14. Outdoor temperature and the measured power data are also provided in the figure. The power measurement includes fan and compressor power of all RTUs and has 5-10 sec sampling time. As mentioned, $\mathrm{PnP}$ and Conv were implemented for the even and odd days, respectively. These results demonstrate consistent demand reduction and improved temperature control for the PnP.

The relative energy savings and peak demand reduction compared to the conventional controller for the one-week experimental period are summarized in Table 5. For the peak demand, the measured power data were summed and a moving average filter with a 15 -min time interval was used in calculating moving average total power. The results show that the PnP achieved about $8 \%$ energy savings and about $43 \%$ peak demand reduction compared to the Conv. The energy savings are due to reduced cycling losses for the PnP control. Because of the unknown internal and external gains and the relatively short experimental period for the comparison, the level of energy savings is less persuasive. However 

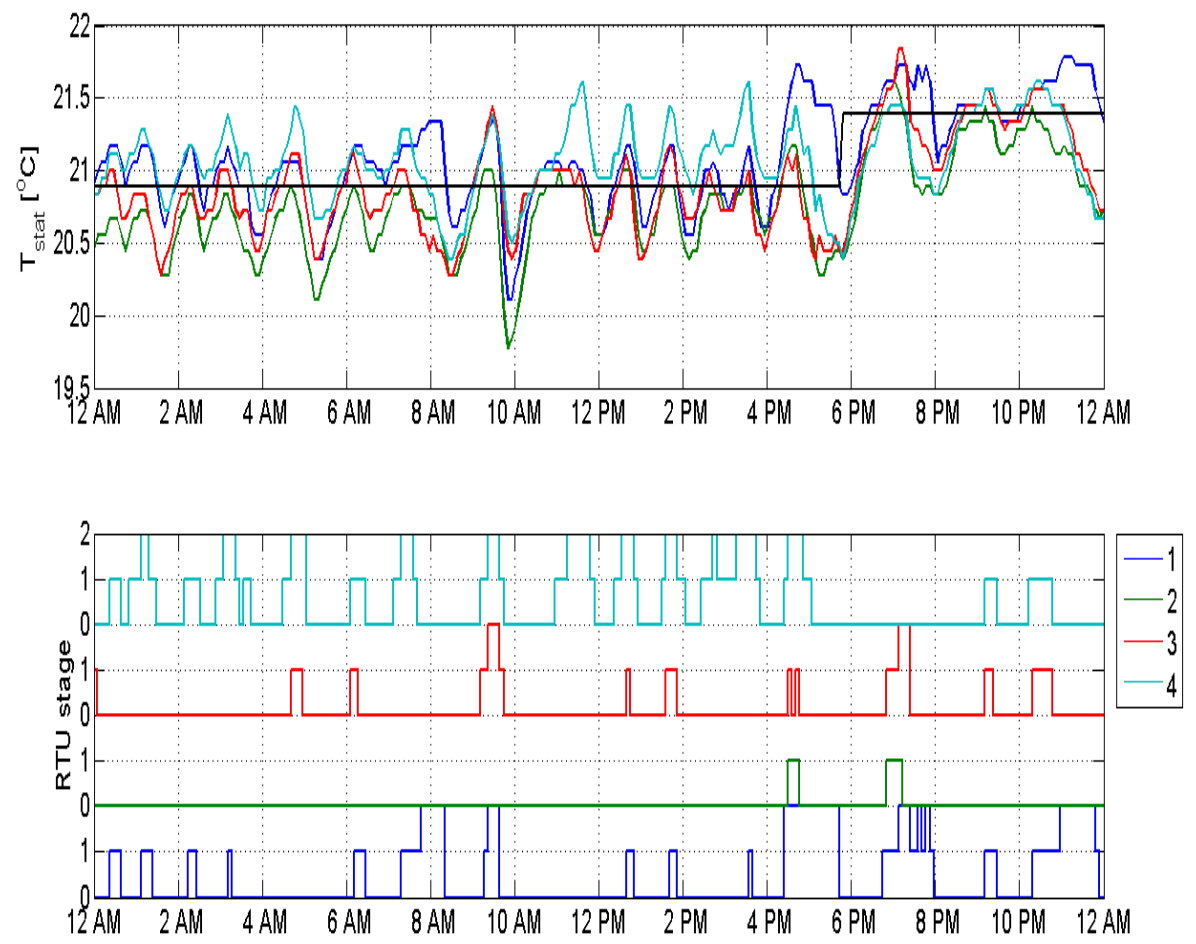

(a) A sample response of a conventional controller (experiment)
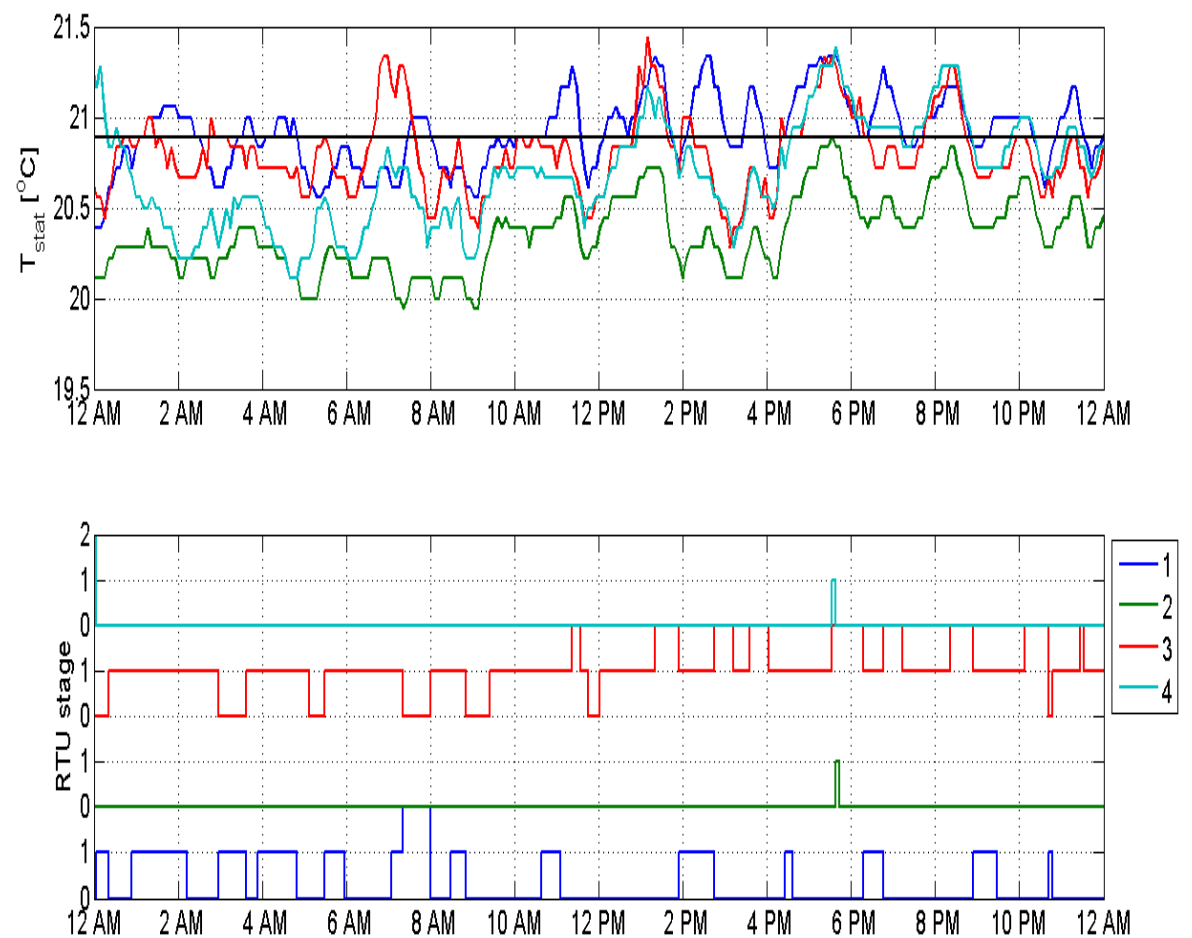

(b) A sample response of $25 \mathrm{PnP}$ controller (experiment)

Figure 13: Controller performance comparisons for experiments at gymnasium 


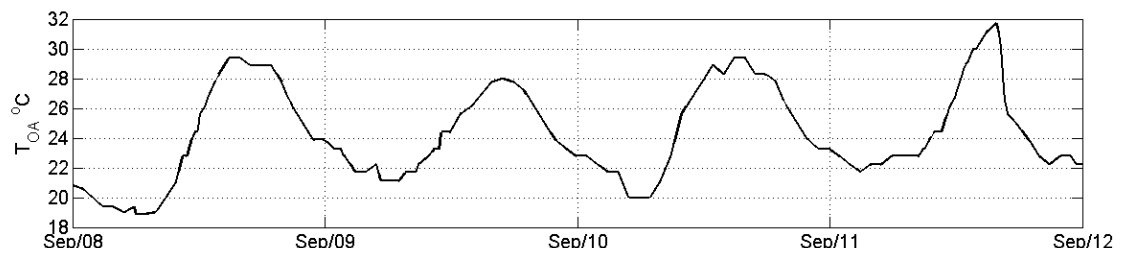

(a) Outdoor air temperature profile
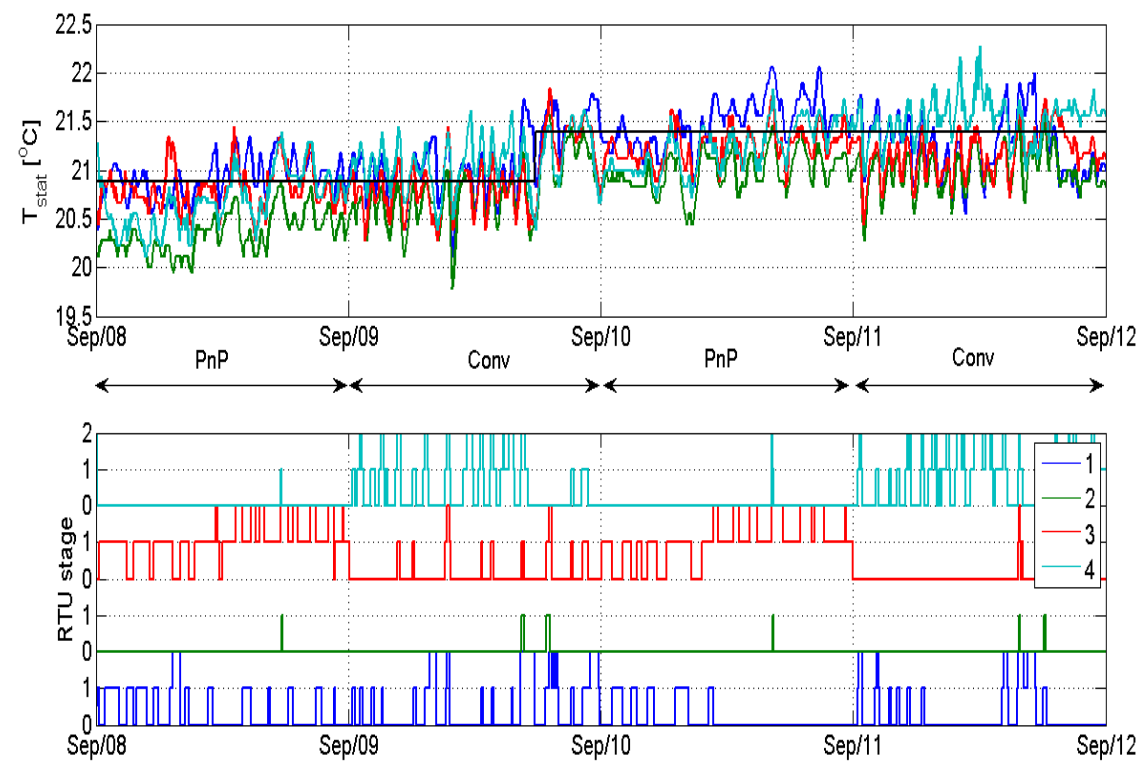

(b) Controller performances

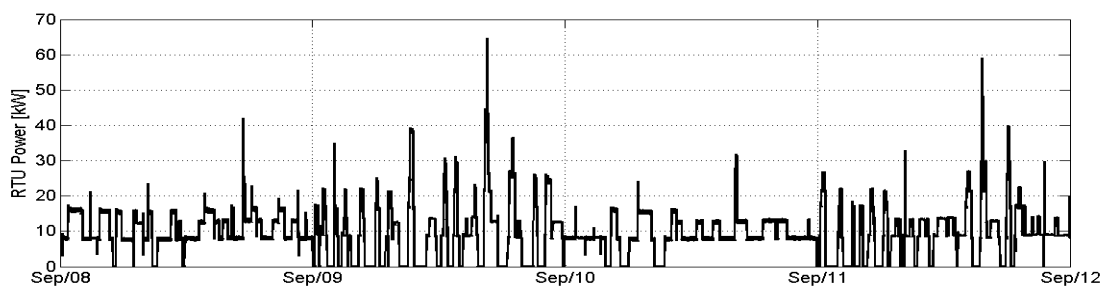

(c) HVAC power profile

Figure 14: Controller performance comparisons for several days at gymnasium 
the peak demand reduction level is appealing even if one takes account of the uncertainty of the gains. In addition to the energy savings and peak demand reduction of the $\mathrm{PnP}$, the $\mathrm{PnP}$ provides better comfort than the Conv. Fig. 15 shows a histogram of the thermostat temperature deviation from the set-point. The right hand side of the origin is the set-point violation. The maximum comfort violations are $1.4^{\circ} \mathrm{C}\left(2.5^{\circ} \mathrm{F}\right)$ and $0.67{ }^{\circ} \mathrm{C}\left(1.2^{\circ} \mathrm{F}\right)$ for the Conv and the $\mathrm{PnP}$ respectively.

Table 5: Relative energy savings and peak demand reduction of PnP relative to Conv for gymnasium case study

\begin{tabular}{|c|c|}
\hline \% Energy savings & \% Peak Demand Reduction \\
\hline 8.23 & $\mathbf{4 2 . 5 7}$ \\
\hline
\end{tabular}
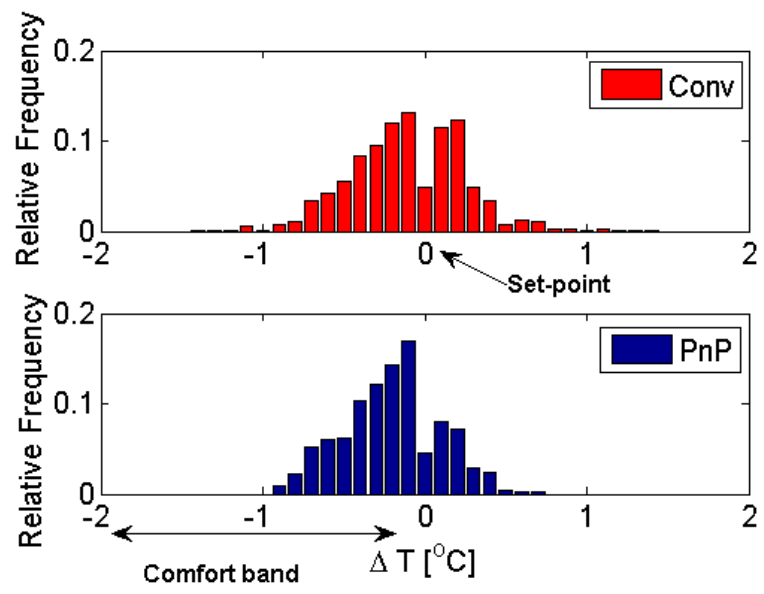

Figure 15: Comparisons of the temperature distributions for one-week experiments at gymnasium

\section{CONCLUSIONS}

Few advanced algorithms for controlling RTUs in small/medium commercial buildings have been developed to date, despite their wide application. We present a practical algorithm for multiple RTU coordination. The modeling approach relies only on thermostat data and was shown through field experiments to provide accurate short-term predictions under the presence of significant disturbances (e.g. solar radiation and variable occupancy) in a building. This reduces the need for expensive additional sensors or data communication systems for control implementation. The proposed control algorithm was tested both within a simulation platform and an existing building (a gymnasium). In 
the simulation, about $20 \%$ building HVAC energy savings and about $30 \%$ peak demand reduction were achieved for a one-month simulation period. For the experiment, about $8 \%$ building energy savings and about $40 \%$ peak demand reduction were achieved. With the gym deployment, 1) no additional sensors were added and 2) once communications functionality were verified, little work was required.

\section{NOMENCLATURE}

RTU roof top unit

PnP controller Plug-and-play RTU coordinator

Conv conventional controller

LS least-squares identification algorithm

RLS recursive least-squares identification algorithm

$\operatorname{ARX}(n a, n b)$ an auto-regressive with exogenous input (ARX) model structure with polynomial degrees of $(n a, n b)$

$y$ controlled variables, the thermostat temperatures

$u$ manipulated variables, the RTU stages

$e(k)$ a mean zero white noise process

$w(k), v(k)$ white noise processes with zero mean

$G_{T}(z)$ the transfer function of the true system

$H_{T}(z)$ the transfer function of the true disturbance representation

$A\left(z^{-1}\right), B\left(z^{-1}\right)$ polynomial matrices

$\hat{y}(k \mid k-1)$ a conditional expectation, i.e. $\hat{y}(k \mid k-1)=E(y(k) \mid y(k-1), y(k-$ $2), \cdots, u(k-1), u(k-2), \cdots)$.

$\mathcal{G}_{k} y(k-1), y(k-2), \cdots, u(k-1), u(k-2), \cdots$

$\epsilon(k)$ an innovation process, i.e. $y(k)-\hat{y}(k \mid k-1)$

$z$ forward time shift operator $z^{-1} x(k)=x(k-1)$ or a complex number for a transfer function

$\theta$ parameters to be estimated

$N$ data length

$\hat{\theta}_{N}$ LS solution with data length $\mathrm{N}$

$(\mathcal{A}, \mathcal{B}, \mathcal{C}, \mathcal{K})$ a state space realization of a ARX model 
$\left(A_{a}, B_{a}, C_{a}, K_{a}\right)$ matrices for an augmented dynamic system

$K_{a}$ Kalman gain for an augmented system

$Q, R$ covariance matrices of the white noise processes, $w, v$

$P$ a solution of algebraic Ricatti equation

$P_{i}$ RTU power of $i^{t h}$ RTU

$P_{\text {tot }}$ total RTU power

$T_{s}$ sampling time

$d$ prediction horizon

$u_{i}$ stage of $i^{\text {th }} \mathrm{RTU}$

$T_{l}, T_{u}$ lower and upper comfort (temperature) bounds

\section{ACKNOWLEDGMENTS}

The authors wish to acknowledge Dr. Phani Teja Kuruganti and Dr. James J. Nutaro for their valuable suggestions. This work was supported by the Department of Energy through the Consortium for Building Energy Innovation.

\section{REFERENCES}

Anderson, B., Johnson, C. R., 1982. Exponential convergence of adaptive identification and control algorithms. Automatica 18 (1), 1-13.

URL http://www.sciencedirect.com/science/article/pii/ 0005109882900218

Anderson, B. D., Moore, J. B., 2012. Optimal filtering. Courier Dover Publications.

Åström, K. J., Wittenmark, B., 2013. Adaptive control. Courier Dover Publications.

Braun, J., 1990. Reducing energy costs and peak electrical demand through optimal control of building thermal storage. ASHRAE transactions 96 (2), $876-888$.

Callier, F. M., Desoer, C., 1991. Linear system theory (springer texts in electrical engineering).

Crawley, D., Lawrie, L., Pedersen, C., Winkelmann, F., 2000. Energy plus: energy simulation program. ASHRAE journal 42 (4), 49-56.

URL http://apps1.eere.energy.gov/buildings/energyplus/ 
Goodwin, G. C., Sin, K. S., 2013. Adaptive filtering prediction and control. Courier Dover Publications.

Johnstone, R. M., Richard Johnson, C., Bitmead, R. R., Anderson, B., 1982. Exponential convergence of recursive least squares with exponential forgetting factor. Systems \& Control Letters 2 (2), 77-82.

URL http://www.sciencedirect.com/science/article/pii/ S0167691182800145

Katipamula, S., Lutes, R., Ngo, H., Underhill, R., 2013. Transactional network platform: Applications.

Kim, D., Braun, J., Cliff, E., Borggaard, J., 2015. Development, validation and application of a coupled reduced-order cfd model for building control applications. Building and Environment.

Kim, D., Cai, J., Braun, J. E., 2014. Comparisons of model structure and identification methods for multiplertu coordination. 9th International Conference on System Simulation in Buildings, Lige.

Klein, S., Beckman, W., Mitchell, J., Duffie, J., Duffie, N., Freeman, T., Mitchell, J., Braun, J., Evans, B., Kummer, J., et al., 2004. Trnsys 16-a transient system simulation program, user manual. Solar Energy Laboratory. Madison: University of Wisconsin-Madison.

Ljung, L. (Ed.), 1999. System Identification (2Nd Ed.): Theory for the User. Prentice Hall PTR, Upper Saddle River, NJ, USA.

Ljung, L., McKelvey, T., 1996. Subspace identification from closed loop data. Signal Processing 52 (2), 209-215.

URL http://www.sciencedirect.com/science/article/pii/ 0165168496000540

Ma, Y., Borrelli, F., Hencey, B., Coffey, B., Bengea, S., Haves, P., 2010. Model predictive control for the operation of building cooling systems. In: American Control Conference (ACC), 2010. IEEE, pp. 5106-5111.

URL http://ieeexplore.iee.org/xpls/abs_all.jsp?arnumber= 5530468

MacGregor, J., Fogal, D., 1995. Closed-loop identification: the role of the noise model and prefilters. Journal of process control 5 (3), 163-171.

URL http://www.sciencedirect.com/science/article/pii/ 095915249597303A 
Maeder, U., Borrelli, F., Morari, M., 2009. Linear offset-free model predictive control. Automatica 45 (10), 2214-2222.

URL http://www.sciencedirect.com/science/article/pii/ S0005109809002969

Mitchell, J. W., Braun, J. E., 2013. Principles of heating, ventilation, and air conditioning in buildings. Wiley.

Muske, K. R., Badgwell, T. A., 2002. Disturbance modeling for offset-free linear model predictive control. Journal of Process Control 12 (5), 617-632.

URL http://www.sciencedirect.com/science/article/pii/ S0959152401000518

Nutaro, J. J., Fugate, D. L., Kuruganti, T., Starke, M. R., Jan 2014. An inexpensive retrofit technology for reducing peak power demand in small and medium commercial buildings. No. 3494. Conference: 3rd International High Performance Buildings Conference at Purdue.

Pannocchia, G., Rawlings, J. B., 2003. Disturbance models for offset-free model-predictive control. AIChE Journal 49 (2), 426-437.

URL http://onlinelibrary.wiley.com/doi/10.1002/aic.690490213/ abstract

Prívara, S., Širokỳ, J., Ferkl, L., Cigler, J., 2011. Model predictive control of a building heating system: The first experience. Energy and Buildings 43 (2), $564-572$.

URL http://www.sciencedirect.com/science/article/pii/ S0378778810003749

Qin, S. J., Badgwell, T. A., 2003. A survey of industrial model predictive control technology. Control engineering practice 11 (7), 733-764.

URL http://www.sciencedirect.com/science/article/pii/ S0967066102001867

Van den Hof, P., 1998. Closed-loop issues in system identification. Annual reviews in control 22, 173-186.

URL http://www.sciencedirect.com/science/article/pii/ S1367578898000169

Wang, W., Katipamula, S., Ngo, H., Underhill, R., Taasevigen, D., Lutes, R., 2013. Advanced rooftop control (arc) retrofit: Field-test results. PNNL-22656, Pacific Northwest National Laboratory. Advertisement formerly in this space. 